LAWRENCE LIVERMORE NAT IO N A L LABORATORY

\section{Far Scrape-Off Layer and Near Wall Plasma Studies in DIII-D}

D.L. Rudakov, J.A Boedo, R.A. Moyer, N.H. Brooks, R.P. Doerner, T.E. Evans, M.E. Fenstermacher, M. Groth, E.M. Hollmann, S. Krashennijov, C.J. Lasnier, M.A. Mahdavi, G.R. McKee, A. McLean, P.C. Stangeby, W.R. Wampler, J.G. Watkins, W.P. West, D.G. Whyte, C.P.C. Wong

December 6, 2004

16th International Conference on Plasma Surface Interactions Portland, ME, United States May 24, 2004 through May 28, 2004 
This document was prepared as an account of work sponsored by an agency of the United States Government. Neither the United States Government nor the University of California nor any of their employees, makes any warranty, express or implied, or assumes any legal liability or responsibility for the accuracy, completeness, or usefulness of any information, apparatus, product, or process disclosed, or represents that its use would not infringe privately owned rights. Reference herein to any specific commercial product, process, or service by trade name, trademark, manufacturer, or otherwise, does not necessarily constitute or imply its endorsement, recommendation, or favoring by the United States Government or the University of California. The views and opinions of authors expressed herein do not necessarily state or reflect those of the United States Government or the University of California, and shall not be used for advertising or product endorsement purposes. 


\title{
Far scrape-off layer and near wall plasma studies in DIII-D
}

\author{
D.L. Rudakov ${ }^{a^{*},}$ J.A. Boedo ${ }^{a}$, R.A. Moyera ${ }^{a}$ N.H. Brooks ${ }^{b}$, R.P. Doernera ${ }^{a}$ T.E. Evans ${ }^{b}$, \\ M.E. Fenstermacher ${ }^{\mathrm{c}}$, M. Groth ${ }^{\mathrm{c}}$, E.M. Hollmann ${ }^{\mathrm{a}}$, S. Krasheninnikova ${ }^{\mathrm{a}}$, C.J. Lasnierc ${ }^{\mathrm{c}}$, M.A. \\ Mahdavi ${ }^{b}$, G.R. McKeed, A. McLean ${ }^{\text {e }}$, P.C. Stangebye, W.R. Wamplerf ${ }^{\mathrm{f}}$, J.G. Watkins ${ }^{\mathrm{f}}$, \\ W.P. West ${ }^{b}$, D.G. Whyted, and C.P.C. Wong ${ }^{b}$ \\ ${ }^{a}$ University of California, San Diego, La Jolla, California 92093-0417, USA \\ ${ }^{b}$ General Atomics, San Diego, California 92186-5608, USA \\ ${ }^{c}$ Lawrence Livermore National Laboratory, Livermore, California 94550, USA \\ ${ }^{d}$ University of Wisconsin, Madison, Wisconsin 53706, USA \\ ${ }^{e}$ University of Toronto Institute for Aerospace Studies, Toronto, M3H 5T6, Canada \\ fSandia National Laboratories, Albuquerque, New Mexico 87185-1129, USA
}

\begin{abstract}
Far scrape-off layer (SOL) plasma parameters in DIII-D depend strongly on the discharge density and confinement regime. In L-mode, cross-field transport increases with the average discharge density and elevates the far SOL density, thus increasing plasma-wall contact. Far SOL density near the low field side (LFS) of the main chamber wall also increases with decreasing plasma current and with decreasing outer wall gap. In $\mathrm{H}$-mode, between edge localized modes (ELMs), plasma-wall contact is weaker than in L-mode. During ELMs plasma fluxes to the LFS wall increase to, or above the L-mode levels. A large fraction of the net cross-field fluxes is convected through the SOL by large amplitude intermittent transport events. In high density L-mode and during ELMs in $\mathrm{H}$-mode, intermittent events propagate all the way to the LFS wall and may cause sputtering.
\end{abstract}


JNM keywords: P0500, P0600

PSI-16 keywords: DIII-D, Edge plasma, Cross-Field Transport, Intermittent transport, Probes

PACS: 52.40.Hf, 52.35.Ra, 52.55.Fa, 52.70.Ds

*Corresponding author address: 9500 Gilman Dr, Mail code 0417, La Jolla, CA 92093-0417

*Corresponding author e-mail: rudakov@fusion.gat.com

Presenting author address: as above

Presenting author e-mail: as above 


\section{Introduction}

Plasma contact with the main chamber wall in a magnetic fusion device should be minimized in order to prevent the damage of the first wall elements by the plasma fluxes and core plasma contamination with impurities. In most modern tokamaks the isolation of the core plasma from the main chamber wall is accomplished by the use of a poloidal divertor magnetic configuration [1], where a magnetic separatrix divides the core plasma from the scrape-off layer (SOL). In an ideal picture of a divertor operation the plasma particles crossing the last closed flux surface (LCFS) into the SOL stream along the open field lines into the divertor volume, where most of the plasma-material interactions (PMI) occur. However, experiments on Alcator C-Mod [2-3], DIII-D [4-6], ASDEX Upgrade [7], JET [8], and other machines have shown that the plasma contact with the main chamber wall in divertor machines may be significant. A number of studies have reported cross-field convective transport of particles and heat in the tokamak SOL $[2-4,6,8]$. This transport, intermittent in time and space, is attributed to coherent structures that are born in the vicinity of the LCFS and propagate towards the wall due to $E \times B$ drifts [9].

The intermittent structures in a tokamak SOL have been studied by Langmuir probes [3-6,8] as well as optical imaging [10]. However, optical measurements are often limited to the "near SOL" region (within 1-2 cm from the LCFS) due to insufficient light emission levels in the far SOL. On the other hand, probes can resolve very low plasma densities, making them suitable for studies of the far SOL regions that are most important for the main wall PMI. In this article we report the studies of the far SOL and near-wall plasmas in DIII-D that rely mostly on the data obtained by the mid-plane reciprocating probe array [4-6].

\section{Dependence of the Far SOL plasma parameters on the discharge conditions}

Figure 1 shows a poloidal cross-section of the DIII-D tokamak with LCFS and a few SOL magnetic flux surfaces for a lower single-null (LSN) discharge. Immediately adjacent to the 
LCFS is the near SOL region where the magnetic field lines connect from the outboard to the inboard side of the torus and terminate at the divertor plates or baffles at both ends. We will refer to this region as "divertor SOL" (DSOL). Radially outwards from the DSOL at the low field side (LFS) of the torus is a region where magnetic field lines terminate at the toroidally symmetric "knee limiter" located at the upper outer side of the vacuum vessel. We will refer to this region, marked by lighter shading in Fig. 1, as "knee limiter shadow" (KLS). Further outwards from the KLS region is the "outer wall shadow" (OWS) region, shown by the darker shaded area in Fig. 1, where the magnetic field lines terminate at the outer wall near the midplane. Any plasma present in the KLS and OWS regions is directly contributing to the PMI at the main chamber wall.

A large set of experimental data was collected over a number of low-power L-mode LSN discharges with the following typical discharge parameters: toroidal magnetic field, $B_{\mathrm{T}}=2 \mathrm{~T}$, plasma current, $I_{\mathrm{p}}=1 \mathrm{MA}$, average plasma density, $\bar{n}_{e}=2.5-5.5 \times 10^{19} \mathrm{~m}^{-3}$. Radial profiles of the SOL plasma density, $n_{\mathrm{e}}$ electron temperature, $T_{\mathrm{e}}$ and normalized density root-meansquare (RMS) fluctuation level, $\tilde{n}_{\mathrm{e}} / n_{\mathrm{e}}$, for four different average discharge densities are shown in Fig. 2. The profiles were generated by averaging the probe data over $1 \mathrm{~ms}$ time intervals and plotting those versus the radial position of the probe. Profile generation process and its implications are discussed in more detail in Ref. [5]. The shading in Fig. 2 corresponds to the convention of Fig. 1. The four density conditions in Fig. 2 correspond to the Greenwald fractions (densities normalized to the Greenwald limit $n_{\mathrm{GW}}$ ), $f_{\mathrm{GW}}$ of $0.27,0.35,0.4$ and 0.5 .

The SOL density profiles show three distinct regions: steep exponential decay in the inner part of DSOL just outside the LCFS; flattening (slower decay) in the outer part of DSOL; faster decay in the KLS region. As $\bar{n}_{\mathrm{e}}$ increases, so does the density decay length in the outer part of the DSOL, and the flattening of the density profile in this region becomes more pronounced. The $T_{\mathrm{e}}$ profiles do not change much with the density. The relative density 
fluctuation profiles stay flat at about 0.3 through most of the DSOL under all density conditions. We should note that the absolute density fluctuation level in the outer part of the DSOL and KLS region does increase with the discharge density.

A number of LSN L-mode discharges had a lower plasma current, $I_{\mathrm{p}}=0.8 \mathrm{MA}$. Profiles of the SOL plasma density for four different values of $\bar{n}_{\mathrm{e}}$ in lower $I_{\mathrm{p}}$ shots are shown in Fig. 3. The density profiles are remarkably different from those in Fig 2. Except for the lowest $\bar{n}_{\mathrm{e}}$, the decay lengths are virtually constant through the SOL up to the OWS boundary. The LCFS densities are comparable to those at higher $I_{\mathrm{p}}$. The most notable difference is observed in the KLS region where the local densities for comparable $\bar{n}_{\mathrm{e}}$ differ by a factor of $2-4$ between the two $I_{\mathrm{p}}$ cases. The $\tilde{n}_{\mathrm{e}} / n_{\mathrm{e}}$ profile (not shown) is similar to that at higher $I_{\mathrm{p}}$, which means that the absolute $n_{e}$ fluctuation levels in the far SOL are higher in the lower $I_{\mathrm{p}}$ case.

There could be a number of explanations for the difference in the far SOL densities between the two $I_{\mathrm{p}}$ cases. In particular, the connection length from the outer mid-plane to the divertor and "knee limiter" is longer for the lower $I_{\mathrm{p}}$ shots. However, the difference is not dramatic, just about 25\%. Still, a higher fluctuation level at lower $I_{\mathrm{p}}$ combined with an increased connection length may result in an increased ratio of the transverse to the parallel particle transport, that in turn can lead to higher far SOL densities. We will present some data substantiating this argument in the next section.

In all the above examples the density profile in the SOL is decaying exponentially in any given region of the SOL, even though the decay lengths may be substantial (comparable to the SOL width). Therefore, an increase in the outer wall gap (OWG) is likely to decrease the main wall plasma contact. This is confirmed by the data shown in Fig. 4, showing the SOL density profiles from the two otherwise similar L-mode shots (with two different $\bar{n}_{\mathrm{e}}$ in each shot) 
with OWG differing by about $2 \mathrm{~cm}$. As expected, the far SOL density is notably higher in the smaller OWG case.

Some of the LSN discharges had spontaneous transitions to H-mode. Fig. 5 shows the radial profiles of the SOL plasma density under $\mathrm{L}$ and $\mathrm{H}$-mode conditions. $\mathrm{H}$-mode data had a few ELMs that show up prominently on the profile. Quite obviously, between the ELMs $n_{\mathrm{e}}$ in the KLS region is well below that in L-mode, while during ELMs it increases up to the L-mode level. Therefore, plasma contact with the main wall in $\mathrm{H}$-mode is lower between the ELMs and comparable during the ELMs to that in L-mode.

\section{Intermittent plasma structures in far SOL}

Previous studies of the cross-field convective transport by intermittent plasma structures in DIII-D have been reported in Refs. [4-6]. The intermittent transport can be a dominant cross-field transport mechanism in the far SOL [5] and is therefore likely to be the key to the difference in the far SOL densities between the two groups of shots in Figs. 2 and 3. This notion is substantiated by Fig. 6 showing $2 \mathrm{~ms}$ portions of the ion saturation current signals from the outer side of the KLS region (corresponding to $R \approx 233 \mathrm{~cm}$ ) in the two highest $\bar{n}_{\mathrm{e}}$ shots from Fig. 2 (lower trace) and Fig. 3 (upper trace). Not only the time-average current is higher by a factor of $\sim 3$ in the lower $I_{\mathrm{p}}$ case, it also features intermittent events that have much larger relative amplitude than those observed in the higher $I_{\mathrm{p}}$ case. Enhanced crossfield transport due to those events can explain the higher far SOL densities in the lower $I_{\mathrm{p}}$ case.

It has been shown that the blobs dissipate particles and heat as they move towards the outer wall [4-6]. However, in high density L-modes and during ELMs in H-mode the blobs can propagate all the way to the outer wall. This is illustrated in Fig. 7 showing the time series of the plasma density and temperature measured by the mid-plane probe in the far SOL in 
high density $\mathrm{L}$-mode $(\mathrm{a}, \mathrm{b})$, and $\mathrm{H}$-mode $(\mathrm{c}, \mathrm{d})$ discharges. Large intermittent events are obvious in all signals, appearing even in the OWL region in the L-mode case. The intermittent spikes in $n_{\mathrm{e}}$ correlate with those in $T_{\mathrm{e}}$ i.e. in high density discharges the intermittent structures feature both densities and temperatures above the ambient conditions even in the far SOL. This may have two consequences: first the intermittent structures can replenish themselves by ionization; and second, their relative contribution to the main wall plasma interactions can be quite significant.

\section{Summary and conclusion}

We have presented experimental evidence that plasma densities and temperatures in DIII-D can be appreciable in the remote SOL regions where the magnetic field lines terminate at the elements of the main chamber wall. Plasma-wall contact increases with the discharge density. Increasing wall gaps can alleviate this problem to some extent. However, even with extended wall gaps, bursts due to intermittent convective transport in high density discharges, particularly during ELMs, are likely to reach the outer wall and cause erosion. This does not present a significant problem for present day tokamaks. Yet, in future devices like ITER with much higher power density and pulse duration, main wall erosion can be a serious concern due to possible wall damage and core plasma contamination with impurities. Therefore, it is essential to better quantify the consequences of the main wall plasma contact in present day tokamaks in order to get reliable predictions and scalings for next generation devices.

\section{Acknowledgment}

Work supported by the U.S. Department of Energy under DE-FG02-04ER54758, DEFC02-04ER54698, *W-7405-ENG-48, and DE-AC04-94AL85000. 


\section{References}

[1] P.C. Stangeby, The Plasma Boundary of Magnetic Fusion Devices, IoP Publishing, 2000.

[2] M.V. Umansky, et al., Phys. Plasmas 5 (1998) 3373.

[3] B. LaBombard, et al., Phys. Plasmas 8 (2001) 2107.

[4] J.A. Boedo, et al., Phys. Plasmas 8 (2001) 4826.

[5] D.L. Rudakov, et al., Plasma Phys. Cont. Fusion 44 (2002), 717.

[6] J.A. Boedo, et al., Phys. Plasmas 10 (2003) 1670.

[7] A. Kallenbach, et al., Nucl. Fusion 43 (2003) 573.

[8] B. Goncalves, et al., Plasma Phys. Control. Fusion 45 (2003) 1627.

[9] S.I. Krasheninnikov, Phys. Lett. A 283 (2001) 368.

[10] S.J. Zweben, et al., Phys. Plasmas 9 (2002) 1981. 


\section{Figure captions}

Fig. 1. Diagnostic arrangement and the structure of the DIII-D low field side SOL in a LSN magnetic configuration.

Fig. 2. SOL profiles of the plasma density (a), electron temperature (b), and relative density fluctuation level (c) in LSN L-mode discharges with $I_{\mathrm{p}}=1 \mathrm{MA}$ and varying average discharge densities, $\bar{n}_{\mathrm{e}}$, of $2.8,3.7,4.3$, and $5.3 \times 10^{19} \mathrm{~m}^{-3}$.

Fig. 3. SOL profiles of the plasma density in LSN L-mode discharges with $I_{p}=0.8 \mathrm{MA}$ and varying $\bar{n}_{\mathrm{e}}$ of $2.8,3.7,4.3$, and $4.5 \times 10^{19} \mathrm{~m}^{-3}$.

Fig. 4. Effect of the outer wall gap change on the far SOL density in LSN L-mode discharges.

Fig. 5. Comparison of the SOL density profiles in LSN L and H-mode discharges.

Fig. 6. Ion saturation current signals in the far SOL $(R=233 \mathrm{~cm})$ in two LSN L-mode discharges with $I_{\mathrm{p}}=1 \mathrm{MA}$ (lower trace) and $I_{\mathrm{p}}=0.8 \mathrm{MA}$ (upper trace) at comparable discharge densities.

Fig. 7. Time traces (plotted versus the radial position of the probe) of the plasma density and electron temperature in the far SOL of high-density $\left(\bar{n}_{e} \approx 6.5 \times 10^{19} \mathrm{~m}^{-3}, f_{G W} \sim 0.6\right)$ L-mode (a,b) and high density $\left(\bar{n}_{e} \approx 1 \times 10^{20} \mathrm{~m}^{-3}, f_{G W} \sim 1\right) \mathrm{H}$-mode (c,d) discharges. 


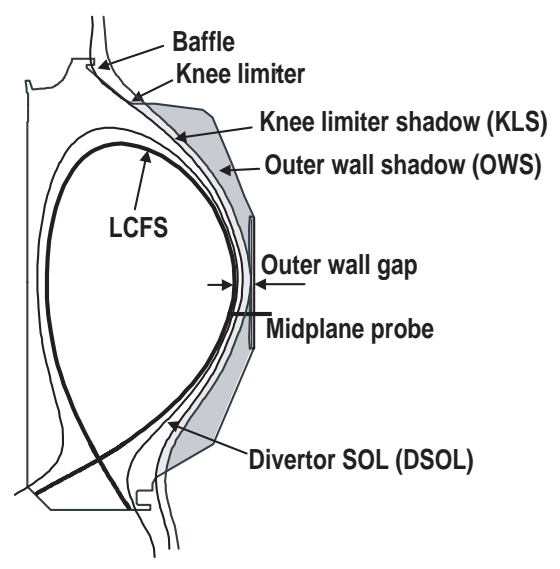

Fig. 1, 0-24, D.L. Rudakov, PSI 2004 


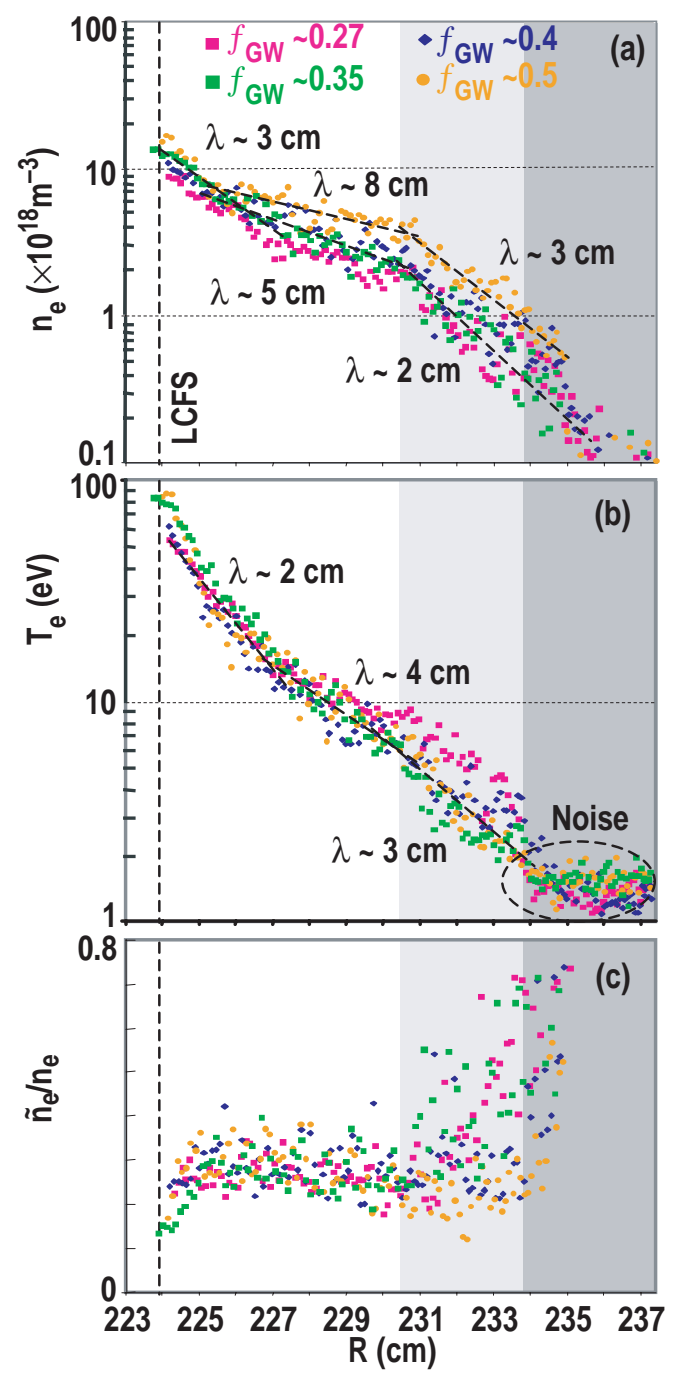

Fig. 2, 0-24, D.L. Rudakov, PSI 2004 


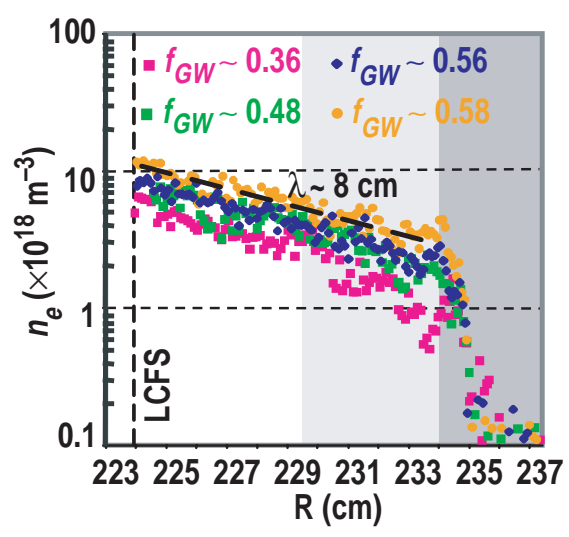

Fig. 3, 0-24, D.L. Rudakov, PSI 2004 


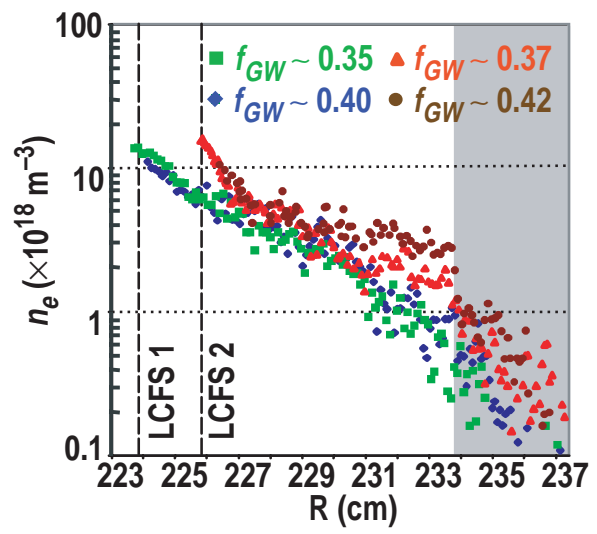

Fig. 4, 0-24, D.L. Rudakov, PSI 2004 


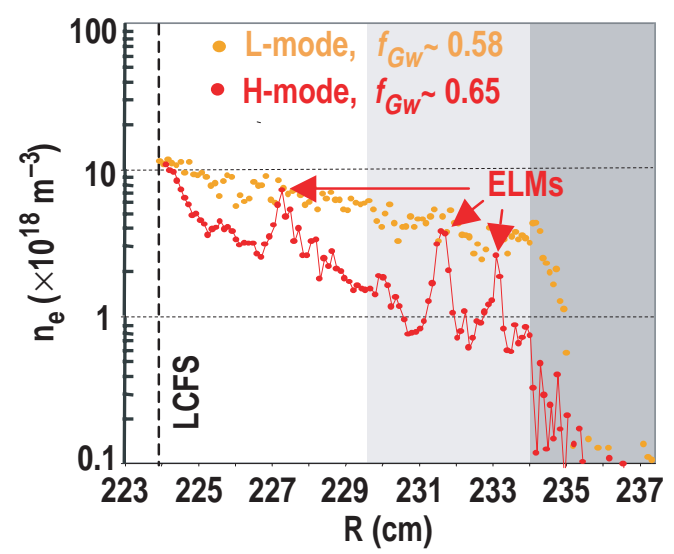

Fig. 5, 0-24, D.L. Rudakov, PSI 2004 


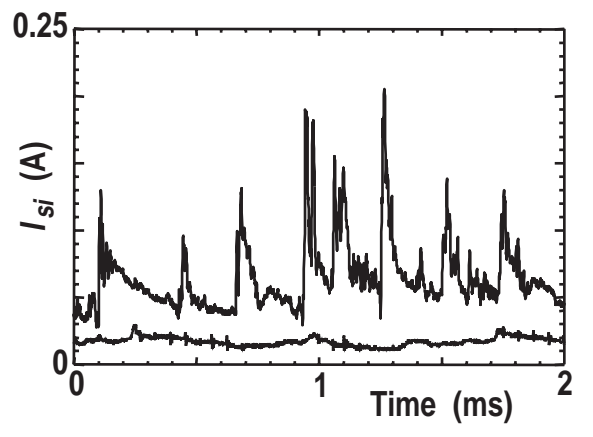

Fig. 6, 0-24, D.L. Rudakov, PSI 2004 


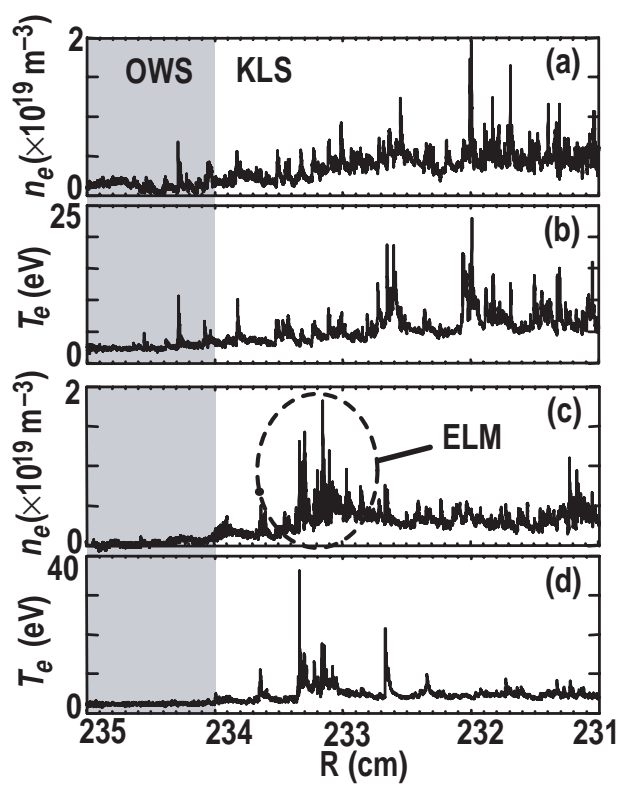

Fig. 7, 0-24, D.L. Rudakov, PSI 2004 